\title{
Math in Economics
}

\author{
Susheng WANG \\ Department of Economics \\ Hong Kong University of Science and Technology
}

December 2014

(C) Hong Kong University of Science \& Technology, 2014 


\section{Contents}

Contents 2

Preface $\quad 6$

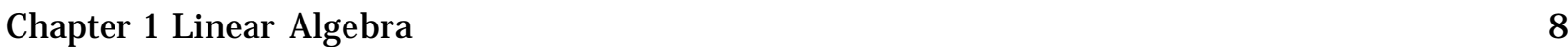

1. Vector 8

2. Matrix 10

3. Linear Equation Systems 16

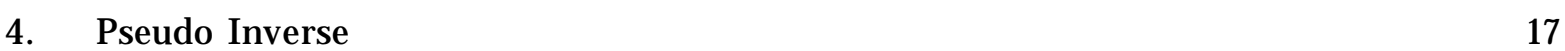

$\begin{array}{llr}\text { 5. } & \text { Elementary Operations } & 18\end{array}$

6. Block Matrix 23

7. Eigenvalue and Diagonalization 24

$\begin{array}{llr}\text { 8. Symmetric Matrix } & 28\end{array}$

9. Idempotent Matrix 30

Notes 31

$\begin{array}{ll}\text { Appendix } & 31\end{array}$

Chapter 2 Basic Real Analysis 37

$\begin{array}{lll}\text { 1. Set } & \text { Set }\end{array}$

2. Logical Statements 40

3. Mappings 41

$\begin{array}{lll}\text { 4. Sequence } & 42\end{array}$

$\begin{array}{lll}\text { 5. Continuity } & 44\end{array}$

$\begin{array}{ll}\text { 6. Differentiation } & 45\end{array}$

$\begin{array}{lll}\text { 7. Directional Differentiation } & 47\end{array}$

8. Homogeneous Function 49

9. Fundamental Theorems 49

$\begin{array}{ll}\text { Notes } & 54\end{array}$

$\begin{array}{ll}\text { Appendix } & 54\end{array}$

Chapter 3 General Optimization $\quad 56$

1. Positive Definite Matrix 56 
2. Concavity 58

$\begin{array}{lll}3 . & \text { Quasi-Concavity } & 63\end{array}$

4. Unconstrained Optimization (Interior Solutions) 69

5. Constrained Optimization (Boundary Solutions) 75

6. Envelope Theorem 85

$\begin{array}{ll}\text { Notes } & 87\end{array}$

$\begin{array}{ll}\text { Appendix } & 88\end{array}$

$\begin{array}{ll}\text { A.1. A Counterexample } & 88\end{array}$

$\begin{array}{ll}\text { A.2. Lemmas } & 90\end{array}$

A.3. Proofs of Propositions 93

A.4. Proofs of Theorems $\quad 94$

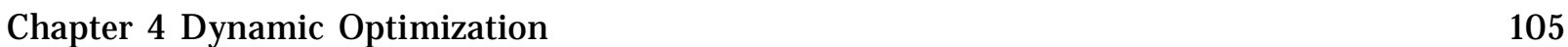

1. Discrete-Time Stochastic Models 106

$\begin{array}{ll}\text { 1.1. An Example } & 106\end{array}$

1.2. Markov Process 109

1.3. Backward Induction 109

$\begin{array}{ll}\text { 1.4. Bellman Equation } & 113\end{array}$

$\begin{array}{ll}\text { 1.5. Lagrange Method } & 117\end{array}$

2. Continuous-Time Deterministic Models 121

2.1. Dynamic Programming: The General Case 122

2.2. Dynamic Programming with State Variables 124

$\begin{array}{ll}\text { 2.3. Transversality Conditions } & 130\end{array}$

2.4. Equivalence between Continuous-Time and Discrete-Time Models 131

3. Phase Diagram 133

3.1. The Linear System 134

3.2. Linearization 141

3.3. Dynamic Path 141

Notes 146

$\begin{array}{ll}\text { Appendix } & 147\end{array}$ 


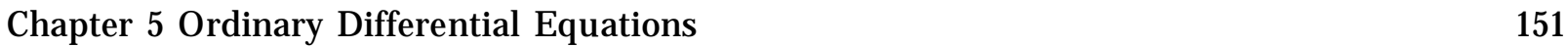

1. An Example 151

$\begin{array}{lll}2 . & \text { Introduction } & 152\end{array}$

3. First-Order Differential Equations 153

3.1. Equations of Separable Variables 153

3.2. Homogeneous Equations 154

3.3. Linear Equations 155

3.4. Exact Equations 156

3.5. The Method of Power Series 159

4. The Method of Laplace Transform 159

5. Linear Equation Systems $\quad 162$

5.1. Existence and Uniqueness 162

5.2. Homogeneous Equations 163

5.3. Linear Equation Systems 165

5.4. Linear Equation Systems with Constant Coefficients 166

$\begin{array}{ll}\text { Notes } & 169\end{array}$

$\begin{array}{ll}\text { Appendix } & 169\end{array}$

Chapter 6 Difference Equations 171

1. Introduction 171

2. $\quad$ First-Order Difference Equations 172

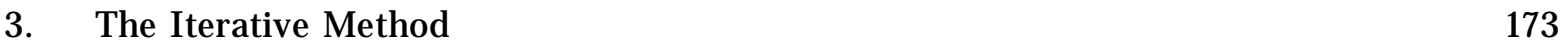

4. The z-Transformation Method 174

$\begin{array}{ll}\text { Notes } & 175\end{array}$

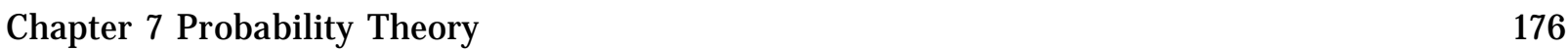

1. Probability Space 176

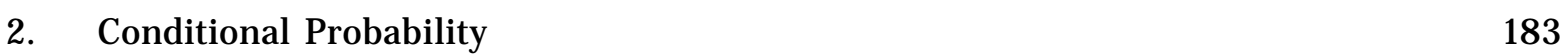

3. Random Variables 190

4. Distribution Functions $\quad 193$

5. Conditional Distributions and Expectations 200 
$\begin{array}{ll}\text { 6. Convergence } & 202\end{array}$

$\begin{array}{ll}\text { Notes } & 204\end{array}$

$\begin{array}{ll}\text { References } & 204\end{array}$

$\begin{array}{ll}\text { Index } & 205\end{array}$ 


\section{Preface}

The target audience. This book covers math at the senior undergraduate, Master's and $\mathrm{PhD}$ levels for students in business schools and economics departments. It concisely covers the main math knowledge and tools useful for business and economics studies, including matrix analysis, basic math concepts, general optimization, dynamic optimization, ordinary differential equations, difference equations, and probability theory. Basic math tools, particularly optimization tools, are essential for students in a business school, especially for students majoring in economics, accounting, finance, management, and marketing. Nowadays most graduate programs in business schools require their students to take a short and intense course in math just before or shortly after commencing their studies. This book is designed to be used in such a course.

The coverage. Chapter 1 focuses on the part of linear algebra that is essential for business studies-matrix analysis. Chapter 2 covers the basic concepts underlying real analysis, including set, sequence, convergence, continuity, differentiation, homogeneity, inverse functions, and fixed points. Chapter 3 discusses general topics in optimization, including definite matrices, concavity, quasi-concavity, unconstrained and constrained optimization, the Lagrange theorem, the Kuhn-Tucker theorem, and the envelope theorem. Chapter 4 explains the concept of dynamic optimization, with a particular emphasis on discrete-time stochastic optimization and continuous-time deterministic optimization. It describes several popular approaches to dynamic optimization, including backward induction, the Lagrange method, the Hamilton method, the Bellman equation, the Euler equation, transversality conditions, and the phase diagram. Chapter 5 covers first-order differential equations and linear equation systems. It introduces various ways of solving first-order differential equations-the standard method of solving linear equations, and the Laplace transformation. Chapter 6 details firstorder difference equations and linear difference equations. It introduces the iterative method and z-transformation. Finally, Chapter 7 covers probability theory, including probability space, conditional probability, random variables, distribution functions, conditional distributions, and convergence.

Special features of this book. There is no shortage of math books for business studies. This book covers all the necessary topics but places a special emphasis on optimization as it is an essential topic for business students. Many details are deliberately left out for conciseness. Thus this book is suitable for those students who will be picking up most of the details in class. It will also appeal to those instructors who prefer to fill in the details themselves. This book can also serve as a handy reference for those who have already learned much of the materials.

Supporting materials. Exercises and solutions are available at www.bm.ust.hk/ -sswang/math-book/. Errors are inevitable and corrections will be posted 
there. Interested readers will also find additional materials such as new sections and chapters at the website. 


\section{Chapter 1 \\ Linear Algebra}

We will focus on the part of linear algebra that is most useful for business studiesmatrices. Basic knowledge of linear algebra is assumed.

\section{Vector}

A scalar is either a real number $a \in \mathbb{R}$ or a complex number $z \in \mathbb{C}$, where $\mathbb{R}$ is the set of all real numbers and $\mathbb{C}$ is the set of all complex numbers. A vector is an ordered sequence of numbers:

$$
x=\left(\begin{array}{c}
x_{1} \\
x_{2} \\
\vdots \\
x_{n}
\end{array}\right)
$$

The numbers $x_{i}$ are called entries, coefficients or elements. The entries can generally be complex numbers. However, except when eigenvalues are involved, we assume real entries and denote an n-dimensional vector by $x \in \mathbb{R}^{n}$. For convenience, we sometimes write it as $\left(x_{i}\right)$ or $\left(x_{i}\right)_{n}$. In business studies, for example, a vector can represent a financial asset, with its entries representing features of the financial asset.

A special vector is the zero vector: $0 \in \mathbb{R}^{n}$. Given a vector in (1.1), we can define its transpose and denote it by $x^{\prime}$ or $x^{T}$, with

$$
x^{\prime}=x^{T} \equiv\left(x_{1}, x_{2}, \ldots, x_{n}\right) .
$$

We typically write a vector as a vertical/column vector; its horizontal/row version is $x^{\prime}$. For a vector $x \in \mathbb{R}^{n}$, we can define its length as $\|x\|$, where

$$
\|x\| \equiv \sqrt{x_{1}^{2}+\cdots+x_{n}^{2}}
$$

and call it the norm. Then we can define the distance between any two points $x$ and $y$ in $\mathbb{R}^{n}$ by the norm $\|x-y\|$.

Given two vectors $a=\left(a_{i}\right)_{n}$ and $b=\left(b_{i}\right)_{n}$, we can define their summation $a+b$, subtraction $a-b$, and multiplication $a^{\prime} b,\langle a, b\rangle$ or $a \cdot b$ respectively by

$$
a+b=\left(\begin{array}{c}
a_{1}+b_{1} \\
\vdots \\
a_{n}+b_{n}
\end{array}\right), \quad a-b=\left(\begin{array}{c}
a_{1}-b_{1} \\
\vdots \\
a_{n}-b_{n}
\end{array}\right), \quad a \cdot b=\sum_{i=1}^{n} a_{i} b_{i} .
$$


We can also multiply a vector $a \in \mathbb{R}^{n}$ by a number $\lambda \in \mathbb{R}$ :

$$
\lambda a \equiv\left(\begin{array}{c}
\lambda a_{1} \\
\vdots \\
\lambda a_{n}
\end{array}\right) .
$$

These operations are intuitively shown in Figure 1.1, and, if $\theta$ is the angle between vectors $a$ and $b$, we have

$$
a \cdot b=\|a\| \cdot\|b\| \cdot \cos (\theta)
$$

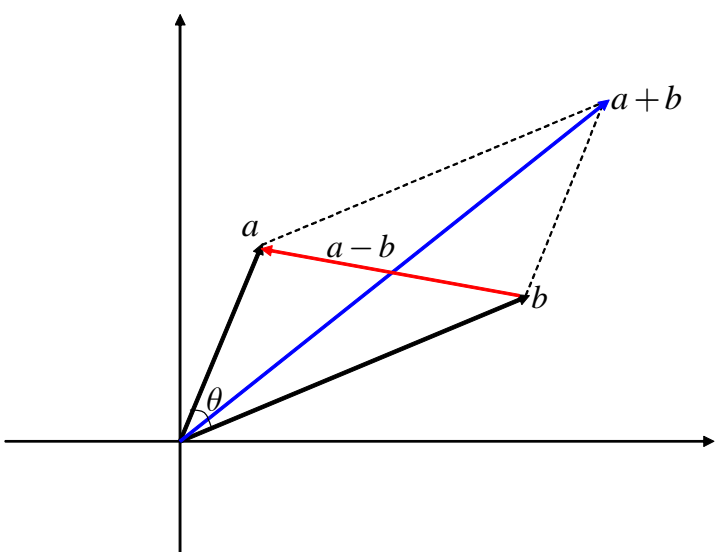

Figure 1.1. Graphic Illustration of Vector Operations

Proposition 1.1. For vectors $a, b, c \in \mathbb{R}^{n}$, we have

(a) the associative law of summation: $(a+b)+c=a+(b+c)$.

(b) the commutative law of summation: $a+b=b+a$.

(c) the commutative law of multiplication: $a \cdot b=b \cdot a$.

(d) the distributive law: $a \cdot(b+c)=a \cdot b+a \cdot c$.

A vector $\beta \in \mathbb{R}^{n}$ is a linear combination of vectors $\alpha_{1}, \ldots, \alpha_{m} \in \mathbb{R}^{n}$, if there exist $\lambda_{1}, \ldots, \lambda_{m} \in$ $\mathbb{R}$ such that

$$
\beta=\lambda_{1} \alpha_{1}+\cdots+\lambda_{m} \alpha_{m} .
$$

For example, a mutual fund is a linear combination of some basic assets. Define the span of a few vectors $\alpha_{1}, \ldots, \alpha_{m}$ as

$$
\operatorname{span}\left(\alpha_{1}, \ldots, \alpha_{m}\right)=\left\{\text { all linear combinations of vectors } \alpha_{1}, \ldots, \alpha_{m}\right\} .
$$

We call $\alpha_{1}, \ldots, \alpha_{m}$ the generating vectors of the span. For example, a financial market can be considered as the span of some basic financial assets.

A few vectors $\alpha_{1}, \alpha_{2}, \ldots, \alpha_{m} \in \mathbb{R}^{n}$ are linearly dependent if there exist numbers $\lambda_{1}, \lambda_{2}, \ldots, \lambda_{m} \in \mathbb{R}$, not all 0 , such that

$$
\lambda_{1} \alpha_{1}+\lambda_{2} \alpha_{2}+\cdots+\lambda_{m} \alpha_{m}=0
$$


Vectors that are not linearly dependent are linearly independent. Hence, vectors $\alpha_{1}, \alpha_{2}, \ldots, \alpha_{m}$ are linearly independent if the equation $c_{1} \alpha_{1}+c_{2} \alpha_{2}+\cdots+c_{m} \alpha_{m}=0$ occurs only if $c_{1}=c_{2}=$ $\cdots=c_{m}=0$. Linear independence means that none of the vectors can be a linear combination of the rest. In $\operatorname{span}\left(\alpha_{1}, \ldots, \alpha_{m}\right)$, if one of the generating vectors, say $\alpha_{m}$, is a linear combination of the rest of the generating vectors, then

$$
\operatorname{span}\left(\alpha_{1}, \ldots, \alpha_{m}\right)=\operatorname{span}\left(\alpha_{1}, \ldots, \alpha_{m-1}\right) .
$$

That is, if a generating vector is a linear combination of other generating vectors, this generating vector is redundant. If we can find a set of linearly independent generating vectors for a space (which we can always do), then the number of such vectors is the minimum possible number of any set of generating vectors for this space.

Example 1.1. Any two linearly independent vectors in $\mathbb{R}^{2}$ can span the whole space $\mathbb{R}^{2}$. This can be shown graphically. In general, $\mathbb{R}^{n}$ can be spanned by $n$ linearly independent vectors.

\section{Matrix}

A matrix is an ordered sequence of column vectors or row vectors, which can be written in the following form:

$$
A=\left(\begin{array}{ccc}
a_{11} & \cdots & a_{1 n} \\
\vdots & & \vdots \\
a_{m 1} & \cdots & a_{m n}
\end{array}\right) .
$$

The numbers $a_{i j}$ are called entries, coefficients or elements. The entries can generally be complex numbers. However, except when eigenvalues are involved, we assume real entries. We say that matrix $A$ in (1.2) is of dimension $m \times n$ and denote $A \in \mathbb{R}^{m \times n}$. For convenience, we sometimes write it as $\left(a_{i j}\right)$ or $\left(a_{i j}\right)_{m \times n}$. In business, a matrix can represent a set of data of a few economic variables.

Example 1.2. The following is a linear equation system for variables $x_{1}, \ldots, x_{n}$ :

$$
\begin{gathered}
a_{11} x_{1}+a_{12} x_{2}+\cdots+a_{1 n} x_{n}=d_{1}, \\
a_{21} x_{1}+a_{22} x_{2}+\cdots+a_{2 n} x_{n}=d_{2}, \\
\vdots \\
a_{m 1} x_{1}+a_{m 2} x_{2}+\cdots+a_{m n} x_{n}=d_{m} .
\end{gathered}
$$

This linear equation system can be written as $A x=d$, where $A$ is defined in (1.2) and $d=$ $\left(d_{i}\right)_{m}$.

A special matrix is the zero matrix: $0 \in \mathbb{R}^{m \times n}$, in which all the entries are zero. When $m=n$, we have a square matrix. A special square matrix is the $\underline{\text { identity matrix: }} I_{n} \in \mathbb{R}^{n \times n}$, in which all the diagonal entries are 1 and the rest are zero. 
Given two matrices $A$ and $B$, we can define their summation $A+B$, subtraction $A-B$, and multiplication $A B$. A requirement for the operations is to have matching dimensions as shown in the following:

$$
A_{m \times n}+B_{m \times n}, \quad A_{m \times n}-B_{m \times n}, \quad A_{m \times n} B_{n \times k} .
$$

For $A=\left(a_{i j}\right)$ and $B=\left(b_{i j}\right)$, with matching dimensions, the operations are defined by

$$
A \pm B=\left(\begin{array}{ccc}
a_{11} \pm b_{11} & \cdots & a_{1 n} \pm b_{1 n} \\
\vdots & & \vdots \\
a_{m 1} \pm b_{m 1} & \cdots & a_{m n} \pm b_{m n}
\end{array}\right), \quad A B=\left(\begin{array}{cccc}
\sum_{t=1}^{n} a_{1 t} b_{t 1} & \cdots & \sum_{k=1}^{n} a_{1 t} b_{t k} \\
\vdots & & \vdots \\
\sum_{t=1}^{n} a_{m t} b_{t 1} & \cdots & \sum_{t=1}^{n} a_{m t} b_{t k}
\end{array}\right)
$$

We can also multiply a matrix $A=\left(a_{i j}\right)_{m \times n}$ by a number $\lambda \in \mathbb{R}$ :

$$
\lambda A \equiv\left(\begin{array}{ccc}
\lambda a_{11} & \cdots & \lambda a_{1 n} \\
\vdots & & \vdots \\
\lambda a_{m 1} & \cdots & \lambda a_{m n}
\end{array}\right) .
$$

Vectors can be treated as one-column matrices, and so the definition of matrix operations applies to vectors too. That is, the operations for one-column matrices are consistent with those defined for vectors.

Example 1.3. Given two vectors $a \in \mathbb{R}^{m}$ and $b \in \mathbb{R}^{n}$, derive $a b^{\prime}$.

Why do we define such matrix operations? The reason is that these definitions turn out to be very convenient in many applications. Take a multiplication operation as an example. Suppose that a vector $x \in \mathbb{R}^{n}$ is mapped linearly to another vector $y \in \mathbb{R}^{n}$ by $y=A x$, where $A \in \mathbb{R}^{n \times n}$, and suppose that the vector $y$ is further mapped to $z \in \mathbb{R}^{n}$ by $z=B y$, where $B \in \mathbb{R}^{n \times n}$. The question is: how can we map $x$ directly to $z$ ? The answer is: we can find a matrix $C \in \mathbb{R}^{n \times n}$ such that $z=C x$ and this matrix $C$ turns out to be $C=B A$, where the multiplication operation is defined above.

Theorem 1.1. Whenever the matrix operations are feasible, we have

(a) the associative law of summation: $(A+B)+C=A+(B+C)$.

(b) the associative law of multiplication: $A(B C)=(A B) C$.

(c) the commutative law of summation: $A+B=B+A$.

(d) the distributive law: $A(B+C)=A B+A C,(B+C) A=B A+C A$.

Denote the determinant of a square matrix $A$ by $|A|$. Why do we denote the determinant this way? The reason is that, for the case of a $3 \times 3$ matrix $A=\left(\alpha_{1}, \alpha_{2}, \alpha_{3}\right)$, the absolute value of the determinant is the size of the object (called parallelepiped) defined by the column vectors of the matrix (see Figure 1.2). 


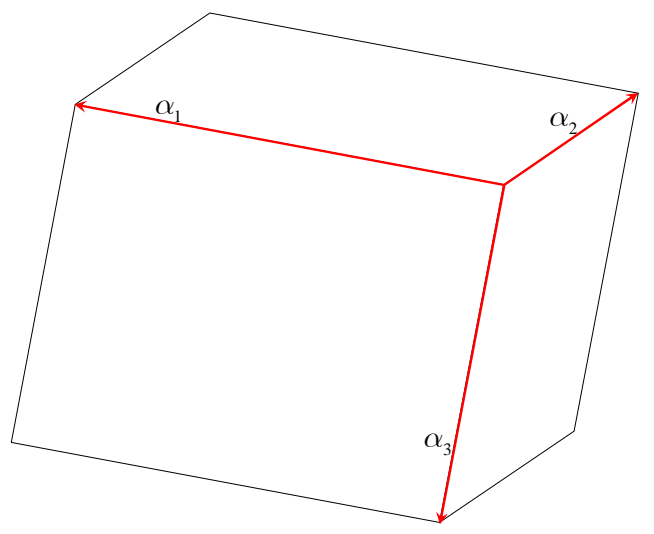

Figure 1.2. The Volume of a Parallelepiped

Theorem 1.2. For any $A, B \in \mathbb{R}^{n \times n}$, we have $|A B|=|A||B|$.

Each $n$-dimensional vector $x$ can be treated as an $n \times 1$ matrix. If so, all the rules for and properties of vectors can be derived from the rules for and properties of matrices. That is, vectors are special matrices.

For $A \in \mathbb{R}^{n \times n}$, if there is another $B \in \mathbb{R}^{n \times n}$ such that $A B=B A=I, A$ is said to be invertible or nonsingular. Then denoting $B$ by $A^{-1}, A^{-1}$ is called the inverse matrix of $A$. The inverse is unique.

How to find the inverse matrix? For this, we need minors. Given a square matrix $A=$ $\left(a_{i j}\right)_{n \times n}$, we call the following determinant the minor of $a_{i j}$ :

$$
M_{i j} \equiv\left|\begin{array}{cccccc}
a_{11} & \cdots & a_{1, j-1} & a_{1, j+1} & \cdots & a_{1 n} \\
\vdots & & \vdots & \vdots & & \vdots \\
a_{i-1,1} & \cdots & a_{i-1, j-1} & a_{i-1, j+1} & \cdots & a_{i-1, n} \\
a_{i+1,1} & \cdots & a_{i+1, j-1} & a_{i+1, j+1} & \cdots & a_{i+1, n} \\
\vdots & & \vdots & \vdots & & \vdots \\
a_{n 1} & \cdots & a_{n, j-1} & a_{n, j+1} & \cdots & a_{n n}
\end{array}\right|,
$$

and call the following value the cofactor of $a_{i j}$ :

$$
C_{i j} \equiv(-1)^{i+j} M_{i j}
$$

Denote

$$
A^{*} \equiv\left(\begin{array}{ccc}
C_{11} & \cdots & C_{1 n} \\
\vdots & & \vdots \\
C_{n 1} & \cdots & C_{n n}
\end{array}\right)^{T}
$$

and call it the adjoint of $A$.

Theorem 1.3. For any square matrix $A=\left(a_{i j}\right) \in \mathbb{R}^{n \times n}$, we have 
(1) $\sum_{k=1}^{n} a_{i k} C_{i k}=\sum_{k=1}^{n} a_{k j} C_{k j}=|A|$, for any $i, j ;$
(2) $\sum_{k=1}^{n} a_{i k} C_{j k}=\sum_{k=1}^{n} a_{k i} C_{k j}=0$, for any $i, j, i \neq j$.

The first result in Theorem 1.3 comes directly from the definition of determinant and the second result can be easily derived from the first result.

Theorem 1.4. For $A \in \mathbb{R}^{n \times n}, A$ is invertible if and only if (iff) $|A| \neq 0$. When $A$ is invertible, the inverse is

$$
A^{-1}=\frac{1}{|A|} A^{*}
$$

Corollary 1.1. For a square matrix $A$,

(1) its inverse matrix is unique;

(2) if there is a matrix $B$ such that $A B=I$ or $B A=I$, then $A$ is invertible and $B=A^{-1}$.

Example 1.4. Find the inverse of

$$
A=\left(\begin{array}{ll}
a & b \\
c & d
\end{array}\right)
$$

We have $C_{11}=d, C_{12}=-c, C_{21}=-b, C_{22}=a$. Thus,

$$
A^{-1}=\frac{1}{a d-b c}\left(\begin{array}{cc}
d & -b \\
-c & a
\end{array}\right) \text {. }
$$

Theorem 1.5. If $A, B \in \mathbb{R}^{n \times n}$ are invertible, then

- $\left(A^{-1}\right)^{-1}=A$,

- $(A B)^{-1}=B^{-1} A^{-1}$.

Given matrix

$$
A=\left(\begin{array}{cccc}
a_{11} & a_{12} & \cdots & a_{1 n} \\
a_{21} & a_{22} & \cdots & a_{2 n} \\
\vdots & \vdots & & \vdots \\
a_{m 1} & a_{m 2} & \cdots & a_{m n}
\end{array}\right)
$$

denote the transpose of $A$ by $A^{\prime}$ or $A^{T}$, where

$$
A^{\prime}=\left(\begin{array}{cccc}
a_{11} & a_{21} & \cdots & a_{m 1} \\
a_{12} & a_{22} & \cdots & a_{m 2} \\
\vdots & \vdots & & \vdots \\
a_{1 n} & a_{2 n} & \cdots & a_{m n}
\end{array}\right) .
$$

That is, $\left(a_{i j}\right)_{m \times n}^{\prime}=\left(a_{j i}\right)_{n \times m}$. We denote the entry of matrix $A$ at position $(i, j)$ by $A_{i j}$, i.e., $A_{i j}=a_{i j}$. Then 


$$
A_{i j}^{\prime}=A_{j i}
$$

A matrix $A$ is symmetric if $A^{\prime}=A$. A symmetric matrix must be a square matrix.

The following theorem presents the key properties of the transpose operation.

Theorem 1.6. For the transpose operation, we have

- $\left(A^{\prime}\right)^{\prime}=A$.

- $(A+B)^{\prime}=A^{\prime}+B^{\prime}$.

- $(A B)^{\prime}=B^{\prime} A^{\prime}$.

- $(c A)^{\prime}=c A^{\prime}$, for any $c \in \mathbb{R}$.

- $\left(A^{\prime}\right)^{-1}=\left(A^{-1}\right)^{\prime}$.

Proof. We prove the third property. We have

$$
(A B)_{i j}^{\prime}=(A B)_{j i}=\sum_{k} A_{j k} B_{k i}=\sum_{k} A_{k j}^{\prime} B_{i k}^{\prime}=\sum_{k} B_{i k}^{\prime} A_{k j}^{\prime}=\left(B^{\prime} A^{\prime}\right)_{i j}
$$

Hence, we have $(A B)^{\prime}=B^{\prime} A^{\prime}$.

We also prove the last property. Since $A^{-1} A=I$, if we take the transpose of both sides, we have $A^{\prime}\left(A^{-1}\right)^{\prime}=I$. This means that $\left(A^{-1}\right)^{\prime}$ is the inverse of $A^{\prime}$, i.e., $\left(A^{-1}\right)^{\prime}=\left(A^{\prime}\right)^{-1}$, which is the last property.

For a square matrix $A \in \mathbb{R}^{n \times n}$, denote

$$
\operatorname{tr}(A) \equiv a_{11}+a_{22}+\cdots+a_{n n}
$$

and call it the trace of $A$. The trace applies only to square matrices. The trace is the second key number of a matrix, following the determinant. The third key number is the rank of a matrix, which will be defined later. The following theorem presents the key properties of the trace.

\section{Theorem 1.7.}

- $\operatorname{tr}(c A)=c \cdot \operatorname{tr}(A)$, for any $c \in \mathbb{R}$.

- $\operatorname{tr}\left(A^{\prime}\right)=\operatorname{tr}(A)$.

- $\operatorname{tr}(A+B)=\operatorname{tr}(A)+\operatorname{tr}(B)$.

- $\operatorname{tr}(A B)=\operatorname{tr}(B A)$.

- $\operatorname{tr}\left(T^{-1} A T\right)=\operatorname{tr}(A)$.

Proof. We prove the fourth property only. We have

$\operatorname{tr}(A B)=\sum_{i}(A B)_{i i}=\sum_{i} \sum_{k} A_{i k} B_{k i}=\sum_{k} \sum_{i} A_{i k} B_{k i}=\sum_{k} \sum_{i} B_{k i} A_{i k}=\sum_{k}(B A)_{k k}=\operatorname{tr}(B A)$.

The last property comes from the fourth property: 


$$
\operatorname{tr}\left(T^{-1} A T\right)=\operatorname{tr}\left(T T^{-1} A\right)=\operatorname{tr}(I A)=\operatorname{tr}(A)
$$

For a matrix $A \in \mathbb{R}^{m \times n}$, if the maximum number of linearly independent row vectors of $A$ is $r$, then $A$ is said to have rank $r$ and is denoted by $\operatorname{rank}(A)=r$. A submatrix of a given matrix consists of a rectangular array of entries in specified rows and columns of the given matrix. These rows and columns need not be adjacent.

Theorem 1.8. For any $A \in \mathbb{R}^{n \times m}$,

$$
\begin{aligned}
\operatorname{rank}(A) & =\text { the maximum number of independent row vectors } \\
& =\text { the maximum number of independent column vectors } \\
& =\text { the size of the largest invertible square submatrix of } A .
\end{aligned}
$$

Example 1.5. Consider matrix $A=\left(\begin{array}{lll}2 & -1 & 3 \\ 4 & -2 & 5 \\ 2 & -1 & 4\end{array}\right)$. Since the first two columns are proportional to each other, there are at most two linearly independent columns. Hence, $\operatorname{rank}(A) \leq 2$. We can easily find a non-singular submatrix:

$$
\left|\begin{array}{ll}
2 & 3 \\
4 & 5
\end{array}\right|=-2 \neq 0
$$

indicating that the first and third columns are linearly independent. Hence, $\operatorname{rank}(A) \geq 2$. Therefore, we must have $\operatorname{rank}(A)=2$.

The following proposition presents some useful properties of the rank.

\section{Proposition 1.2.}

- For $A \in \mathbb{R}^{m \times n}, B \in \mathbb{R}^{n \times k}$,

$$
\operatorname{rank}(A)+\operatorname{rank}(B)-n \leq \operatorname{rank}(A B) \leq \min [\operatorname{rank}(A), \operatorname{rank}(B)] .
$$

- For $A \in \mathbb{R}^{m \times n}, B \in \mathbb{R}^{m \times n}$,

$$
\operatorname{rank}(A+B) \leq \operatorname{rank}(A)+\operatorname{rank}(B) .
$$

Another definition of a matrix product is sometimes useful too. Given two matrices $A$ and $B$, the Kronecker product is

$$
A \otimes B=\left(\begin{array}{ccc}
a_{11} B & \cdots & a_{1 n} B \\
\vdots & & \vdots \\
a_{m 1} B & \cdots & a_{m n} B
\end{array}\right), \quad \text { where } A=\left(\begin{array}{ccc}
a_{11} & \cdots & a_{1 n} \\
\vdots & & \vdots \\
a_{m 1} & \cdots & a_{m n}
\end{array}\right)
$$

Example 1.6.

$$
\left(\begin{array}{ll}
3 & 0 \\
5 & 2
\end{array}\right) \otimes\left(\begin{array}{ll}
1 & 4 \\
4 & 7
\end{array}\right)=\left(\begin{array}{ccc}
3\left(\begin{array}{ll}
1 & 4 \\
4 & 7
\end{array}\right) & 0\left(\begin{array}{ll}
1 & 4 \\
4 & 7
\end{array}\right) \\
5\left(\begin{array}{ll}
1 & 4 \\
4 & 7
\end{array}\right) & 7\left(\begin{array}{ll}
1 & 4 \\
4 & 7
\end{array}\right)
\end{array}\right)=\left(\begin{array}{cccc}
3 & 12 & 0 & 0 \\
12 & 21 & 0 & 0 \\
5 & 20 & 7 & 28 \\
20 & 35 & 28 & 49
\end{array}\right)
$$


The following theorem presents the key properties of the Kronecker product.

\section{Theorem 1.9.}

- $(A \otimes B)^{-1}=A^{-1} \otimes B^{-1}$.

- $\quad(A \otimes B)^{\prime}=A^{\prime} \otimes B^{\prime}$.

- $|A \otimes B|=|A|^{m}|B|^{n}$, if $A \in \mathbb{R}^{n \times n}$ and $B \in \mathbb{R}^{m \times m}$.

- $\operatorname{tr}(A \otimes B)=\operatorname{tr}(A) \cdot \operatorname{tr}(B)$.

\section{Linear Equation Systems}

For a matrix $A \in \mathbb{R}^{m \times n}$ and a vector $d \in \mathbb{R}^{m}$, the following $m$ equation for the vector $x \in \mathbb{R}^{n}$ is called a linear equation system:

$$
A x=d
$$

We call the following equation the homogeneous linear system of (1.3):

$$
A x=0 .
$$

The following theorem finds a condition for its existence.

Theorem 1.10. Equation (1.3) has a solution iff $\operatorname{rank}(A)=\operatorname{rank}(A, d)$.

Theorem 1.11 (Cramer's Rule). Given a square matrix $A \in \mathbb{R}^{n \times n}$ and writing $A$ as a sequence of column vectors $A=\left(a_{1}, a_{2}, \ldots, a_{n}\right)$, if $A$ is nonsingular, then (1.3) has a unique solution and it is $\bar{x}=\left(\bar{x}_{1}, \ldots, \bar{x}_{n}\right)^{\prime}$, where

$$
\bar{x}_{i}=\frac{\left|a_{1}, a_{2}, \ldots, a_{i-1}, d, a_{i+1}, \ldots a_{n}\right|}{|A|}, \quad i=1,2, \ldots, n .
$$

Example 1.7. Solve the following equation system:

$$
\left\{\begin{array}{l}
2 x_{1}+3 x_{2}=4 \\
3 x_{1}-5 x_{2}=1
\end{array}\right.
$$

By Cramer's Rule, we can quickly find the solution:

$$
x_{1}=\frac{\left|\begin{array}{cc}
4 & 3 \\
1 & -5
\end{array}\right|}{\left|\begin{array}{cc}
2 & 3 \\
3 & -5
\end{array}\right|}=\frac{23}{19}, \quad x_{2}=\frac{\left|\begin{array}{cc}
2 & 4 \\
3 & 1
\end{array}\right|}{\left|\begin{array}{cc}
2 & 3 \\
3 & -5
\end{array}\right|}=\frac{10}{19}
$$

Let $N(A)$ be the set of all solutions of (1.4). Call $N(A)$ the null space of $A$ or the solution set of (1.4). 
Theorem 1.12. If $\operatorname{rank}(A)=r$,

- and if $r=n$, then (1.4) has a unique solution and (1.3) has either a unique solution or no solution.

- There exist $n-r$ linearly independent solutions $\alpha_{1}, \ldots, \alpha_{n-r}$ of (1.4) such that $N(A)=$ $\operatorname{span}\left(\alpha_{1}, \ldots, \alpha_{n-r}\right)$.

- Suppose that $x^{*}$ is a solution of (1.3). Then $x$ is a solution of (1.3) iff there is $x_{0} \in N(A)$ such that $x=x^{*}+x_{0}$.

The rank indicates the number of independent equations in (1.4). Theorem 1.12 is intuitive, and indicates that if $r$ equations in (1.4) are independent, the equation system in (1.4) has $n-r$ degrees of freedom. Further, if a solution of (1.3) exists, then this degree of freedom applies to (1.3) as well.

\section{Pseudo Inverse}

A matrix $A \in \mathbb{R}^{m \times n}$ is a tall matrix if $m>n$. A matrix $A \in \mathbb{R}^{m \times n}$ has full rank if it has the largest possible rank: $\operatorname{rank}(A)=\min (n, m)$.

The inverse matrix is defined only for a nonsingular square matrix. The pseudo inverse extends the existence of an inverse matrix to any matrix. Specifically, for an arbitrary matrix $A \in \mathbb{R}^{m \times n}$, a matrix $A^{+}$is called the pseudo inverse of $A$ if

- $A A^{+} A=A$ and $A^{+} A A^{+}=A^{+}$, and

- $A^{+} A$ and $A A^{+}$are symmetric.

\section{Theorem 1.13.}

(a) For any matrix $A$, there is a unique pseudo inverse $A^{+}$.

(b) If $A$ is nonsingular, then $A^{+}=A^{-1}$.

(c) If $A$ is a tall matrix with full rank, then $A^{+}=\left(A^{\prime} A\right)^{-1} A^{\prime}$.

Part (a) of Theorem 1.13 indicates that every matrix has a pseudo inverse. See Greene (1993, p.37) for the construction of $A^{+}$. Parts (b) and (c) can be easily verified.

Consider an over-determined system of equations:

$$
A x=d,
$$

where $A$ is a tall matrix. If $A$ has full rank, then the solution is $x=A^{+} d$. We can see this easily. Since $A^{\prime} A x=A^{\prime} d$, we immediately have $x=A^{+} d$. This solution is the so-called least-squares solution. 


\section{Elementary Operations}

Elementary operations are excellent for manipulating matrices. Elementary row operations on a matrix $A$ include:

1. Multiplying a row of $A$ by a non-zero scalar $k$.

$$
\text { e.g., } \quad\left(\begin{array}{ll}
a_{11} & a_{12} \\
a_{21} & a_{22}
\end{array}\right) \rightarrow\left(\begin{array}{cc}
k a_{11} & k a_{12} \\
a_{21} & a_{22}
\end{array}\right) \text {. }
$$

2. Multiplying a row of $A$ by a scalar $k$ and adding it to another row.

$$
\text { e.g., } \quad\left(\begin{array}{ll}
a_{11} & a_{12} \\
a_{21} & a_{22}
\end{array}\right) \rightarrow\left(\begin{array}{cc}
a_{11} & a_{12} \\
a_{21}+k a_{11} & a_{22}+k a_{12}
\end{array}\right) .
$$

3. Interchanging two rows of $A$.

$$
\text { e.g., } \quad\left(\begin{array}{ll}
a_{11} & a_{12} \\
a_{21} & a_{22}
\end{array}\right) \rightarrow\left(\begin{array}{ll}
a_{21} & a_{22} \\
a_{11} & a_{12}
\end{array}\right) \text {. }
$$

Elementary column operations are similarly defined. An elementary operation is either an elementary row operation or an elementary column operation. Two matrices $A$ and $B$ are rowequivalent if one can be obtained from the other by a finite sequence of elementary row operations.

Elementary operations have many applications. One of them is to solve a linear equation system. The following theorem outlines the method.

Theorem 1.14. For $A, B \in \mathbb{R}^{m \times n}$ and $c, d \in \mathbb{R}^{m}$, if $(A, d)$ and $(B, c)$ are row-equivalent, then the linear systems $A x=d$ and $B x=c$ have the same solutions.

The proof of the above theorem is straightforward. The theorem, however, provides a convenient way to solve linear systems. The following three examples show the method.

Example 1.8. For a system of equations,

$$
\left(\begin{array}{cccc}
1 & -2 & 3 & -4 \\
0 & 1 & -1 & 1 \\
1 & 3 & 0 & 1 \\
0 & -7 & 3 & 1
\end{array}\right) x=\left(\begin{array}{c}
4 \\
-3 \\
1 \\
-3
\end{array}\right)
$$

we do the following elementary row operations: 


$$
\begin{aligned}
& \left(\begin{array}{ccccc}
1 & -2 & 3 & -4 & 4 \\
0 & 1 & -1 & 1 & -3 \\
1 & 3 & 0 & 1 & 1 \\
0 & -7 & 3 & 1 & -3
\end{array}\right) \rightarrow\left(\begin{array}{ccccc}
1 & -2 & 3 & -4 & 4 \\
0 & 1 & -1 & 1 & -3 \\
0 & 5 & -3 & 5 & -3 \\
0 & -7 & 3 & 1 & -3
\end{array}\right) \rightarrow\left(\begin{array}{ccccc}
1 & -2 & 3 & -4 & 4 \\
0 & 1 & -1 & 1 & -3 \\
0 & 0 & 2 & 0 & 12 \\
0 & 0 & -4 & 8 & -24
\end{array}\right) \rightarrow \\
& \left(\begin{array}{ccccc}
1 & -2 & 3 & -4 & 4 \\
0 & 1 & -1 & 1 & -3 \\
0 & 0 & 1 & 0 & 6 \\
0 & 0 & -1 & 2 & -6
\end{array}\right) \rightarrow\left(\begin{array}{ccccc}
1 & -2 & 3 & -4 & 4 \\
0 & 1 & -1 & 1 & -3 \\
0 & 0 & 1 & 0 & 6 \\
0 & 0 & 0 & 2 & 0
\end{array}\right) \rightarrow\left(\begin{array}{ccccc}
1 & -2 & 3 & -4 & 4 \\
0 & 1 & -1 & 1 & -3 \\
0 & 0 & 1 & 0 & 6 \\
0 & 0 & 0 & 1 & 0
\end{array}\right) \rightarrow \\
& \left(\begin{array}{ccccc}
1 & -2 & 3 & 0 & 4 \\
0 & 1 & -1 & 0 & -3 \\
0 & 0 & 1 & 0 & 6 \\
0 & 0 & 0 & 1 & 0
\end{array}\right) \rightarrow\left(\begin{array}{ccccc}
1 & -2 & 0 & 0 & -14 \\
0 & 1 & 0 & 0 & 3 \\
0 & 0 & 1 & 0 & 6 \\
0 & 0 & 0 & 1 & 0
\end{array}\right) \rightarrow\left(\begin{array}{ccccc}
1 & 0 & 0 & 0 & -8 \\
0 & 1 & 0 & 0 & 3 \\
0 & 0 & 1 & 0 & 6 \\
0 & 0 & 0 & 1 & 0
\end{array}\right) .
\end{aligned}
$$

Hence, the solution is

$$
x_{1}=-8, \quad x_{2}=3, \quad x_{3}=6, \quad x_{4}=0 .
$$

Example 1.9. Consider

$$
A x=0
$$

where

$$
A=\left(\begin{array}{lll}
2 & -1 & 3 \\
4 & -2 & 5 \\
2 & -1 & 4
\end{array}\right)
$$

Do the following elementary row operations:

$$
\left(\begin{array}{lll}
2 & -1 & 3 \\
4 & -2 & 5 \\
2 & -1 & 4
\end{array}\right) \rightarrow\left(\begin{array}{ccc}
2 & -1 & 3 \\
0 & 0 & -1 \\
0 & 0 & 1
\end{array}\right) \rightarrow\left(\begin{array}{ccc}
2 & -1 & 0 \\
0 & 0 & 0 \\
0 & 0 & 1
\end{array}\right)
$$

That is, the original system of equations is equivalent to

$$
\left\{\begin{array}{l}
2 x_{1}-x_{2}=0 \\
x_{3}=0
\end{array}\right.
$$

The solution is therefore

$$
\left\{\begin{array}{l}
x_{1}=\frac{1}{2} x_{2} \\
x_{3}=0
\end{array}\right.
$$

where $x_{2}$ is arbitrary. This means that the null space $N(A)$ has one degree of freedom or the dimension of $N(A)$ is 1 . More precisely,

$$
N(A)=\left\{k\left(\begin{array}{l}
1 \\
2 \\
0
\end{array}\right) \mid k \in \mathbb{R}\right\}
$$

which is a one-dimensional subspace generated by the vector $(1,2,0)^{\prime}$. Example 1.5 has shown that matrix $A$ has rank 2. By Theorem 1.12, we expect its solution set $N(A)$ to be of dimension one, which is consistent with our solution in (1.5). 
Example 1.10. Consider

$$
A x=0,
$$

where

$$
A=\left(\begin{array}{lll}
2 & -1 & 1 \\
4 & -2 & 2 \\
2 & -1 & 1
\end{array}\right)
$$

This matrix can be transformed into the following matrix by elementary row operations:

$$
\left(\begin{array}{ccc}
2 & -1 & 1 \\
0 & 0 & 0 \\
0 & 0 & 0
\end{array}\right)
$$

We can easily find two solutions,

$$
\alpha_{1}=\left(\begin{array}{l}
0 \\
1 \\
1
\end{array}\right), \quad \alpha_{2}=\left(\begin{array}{l}
1 \\
2 \\
0
\end{array}\right)
$$

We have intentionally found two linearly independent solutions. Hence, by Theorem 1.12, we have $N(A)=\operatorname{span}\left(\alpha_{1}, \alpha_{2}\right)$.

Example 1.11. We now solve the following equation system:

$$
\left(\begin{array}{lll}
2 & -1 & 3 \\
4 & -2 & 5 \\
2 & -1 & 4
\end{array}\right) x=\left(\begin{array}{l}
1 \\
1 \\
2
\end{array}\right)
$$

Using elementary row operations, we have

$$
\left(\begin{array}{cccc}
2 & -1 & 3 & 1 \\
4 & -2 & 5 & 1 \\
2 & -1 & 4 & 2
\end{array}\right) \rightarrow\left(\begin{array}{cccc}
2 & -1 & 3 & 1 \\
0 & 0 & -1 & -1 \\
0 & 0 & 1 & 1
\end{array}\right) \rightarrow\left(\begin{array}{cccc}
2 & -1 & 0 & -2 \\
0 & 0 & 0 & 0 \\
0 & 0 & 1 & 1
\end{array}\right)
$$

which implies the following equation system:

$$
\left\{\begin{array}{l}
2 x_{1}-x_{2}=-2 \\
x_{3}=1
\end{array}\right.
$$

One solution of this equation is $x^{*} \equiv(-1,0,1)$, which is one particular solution of (1.6). The solution set of the homogeneous equation of (1.6) is $N(A)$ defined in (1.5). Hence, the general solution of (1.6) is $x=x^{*}+x_{0}$, where $x_{0} \in N(A)$.

Let $e$ be an elementary operation. Denote the resulting matrix after the operation $e$ on $A$ by $e(A)$. For the identity matrix, we call $e(I)$ an elementary matrix. We can easily verify the following proposition.

\section{Theorem 1.15.}

- Elementary matrices are invertible.

- If $e$ is an elementary row operation, then $e(A)=e(I) A$. 
- If $e$ is an elementary column operation, then $e(A)=A e(I)$.

We verify Theorem 1.15 in the following example.

Example 1.12. Suppose that $e_{2}$ is a second type of row operation that multiplies the first row by $k$ and adds it to the second row:

$$
e_{2}\left[\left(\begin{array}{ll}
a_{11} & a_{12} \\
a_{21} & a_{22}
\end{array}\right)\right]=\left(\begin{array}{cc}
a_{11} & a_{12} \\
a_{21}+k a_{11} & a_{22}+k a_{12}
\end{array}\right) .
$$

We have

$$
e_{2}(I)=\left(\begin{array}{ll}
1 & 0 \\
k & 1
\end{array}\right)
$$

and

$$
e_{2}(I)\left(\begin{array}{ll}
a_{11} & a_{12} \\
a_{21} & a_{22}
\end{array}\right)=\left(\begin{array}{cc}
a_{11} & a_{12} \\
a_{21}+k a_{11} & a_{22}+k a_{12}
\end{array}\right)
$$

Therefore,

$$
e_{2}\left[\left(\begin{array}{ll}
a_{11} & a_{12} \\
a_{21} & a_{22}
\end{array}\right)\right]=e_{2}(I)\left(\begin{array}{ll}
a_{11} & a_{12} \\
a_{21} & a_{22}
\end{array}\right)
$$

This verifies Theorem 1.15 for this special case.

The inverse operation of an elementary operation is an elementary operation that brings the matrix back to its original state.

Proposition 1.3. The inverse operation of an elementary row operation exists and is an elementary row operation of the same type.

We show Proposition 1.3 by the following example.

Example 1.13. Let us now see if an inverse operation of $e_{2}$ in Example 1.12 exists. Define an elementary row operation $\tilde{e}_{2}$ by

$$
\tilde{e}_{2}(I) \equiv\left(\begin{array}{cc}
1 & 0 \\
-k & 1
\end{array}\right)
$$

We have

$$
\tilde{e}_{2}(I)\left(\begin{array}{cc}
a_{11} & a_{12} \\
a_{21}+k a_{11} & a_{22}+k a_{12}
\end{array}\right)=\left(\begin{array}{ll}
a_{11} & a_{12} \\
a_{21} & a_{22}
\end{array}\right) .
$$

That is, $\tilde{e}_{2}$ is an inverse operation of $e_{2}$. We can see that $\tilde{e}_{2}$ is also a second type of row operation. This verifies Proposition 1.3 for this special case. Notice that

$$
\tilde{e}_{2}(I) e_{2}(I)=I,
$$

implying $\tilde{e}_{2}(I)=\left[e_{2}(I)\right]^{-1}$. 
Elementary operations can also be used to find an inverse matrix. The following theorem outlines the method.

Theorem 1.16. For $A, B \in \mathbb{R}^{n \times n}$, if there is a sequence of elementary row operations converting $(A, I)$ to $(I, B)$, then $B=A^{-1}$.

With Theorem 1.16, we demonstrate the second application of elementary operations: we can use row elementary operations to find an inverse matrix.

Example 1.14. Find the inverse matrix of the following matrix:

$$
A=\left(\begin{array}{ccc}
0 & 1 & 2 \\
1 & 1 & 4 \\
2 & -1 & 0
\end{array}\right)
$$

Do the following elementary row operations:

$$
\begin{aligned}
&\left(\begin{array}{cccccc}
0 & 1 & 2 & 1 & & \\
1 & 1 & 4 & & 1 & \\
2 & -1 & 0 & & 1
\end{array}\right) \rightarrow\left(\begin{array}{ccccccc}
1 & 1 & 4 & 0 & 1 & 0 \\
0 & 1 & 2 & 1 & 0 & 0 \\
2 & -1 & 0 & 0 & 0 & 1
\end{array}\right) \rightarrow\left(\begin{array}{cccccc}
1 & 1 & 4 & 0 & 1 & 0 \\
0 & 1 & 2 & 1 & 0 & 0 \\
0 & -3 & -8 & 0 & -2 & 1
\end{array}\right) \rightarrow \\
&\left(\begin{array}{ccccccc}
1 & 1 & 4 & 0 & 1 & 0 \\
0 & 1 & 2 & 1 & 0 & 0 \\
0 & 0 & -2 & 3 & -2 & 1
\end{array}\right) \rightarrow\left(\begin{array}{ccccccc}
1 & 1 & 0 & 6 & -3 & 2 \\
0 & 1 & 0 & 4 & -2 & 1 \\
0 & 0 & -2 & 3 & -2 & 1
\end{array}\right) \rightarrow\left(\begin{array}{cccccc}
1 & 0 & 0 & 2 & -1 & 1 \\
0 & 1 & 0 & 4 & -2 & 1 \\
0 & 0 & -2 & 3 & -2 & 1
\end{array}\right) .
\end{aligned}
$$

Therefore,

$$
A^{-1}=\left(\begin{array}{ccc}
2 & -1 & 1 \\
4 & -2 & 1 \\
-\frac{3}{2} & 1 & -\frac{1}{2}
\end{array}\right)
$$

The above derivation suggests how to prove the following theorem.

Theorem 1.17. Any invertible matrix $A \in \mathbb{R}^{n \times n}$ is row-equivalent to the identity matrix.

Theorem 1.17 guarantees that the method of finding an inverse matrix using elementary operations will always work as long as the inverse matrix exists.

The third application of elementary operations is to find the rank of a matrix. For this purpose, we present the following theorem.

Theorem 1.18. The rank of any matrix will not change when multiplied by a nonsingular matrix. That is, for $A \in \mathbb{R}^{m \times n}, P \in \mathbb{R}^{m \times m}$, and $Q \in \mathbb{R}^{n \times n}$, if $P$ and $Q$ are invertible, then

$$
\operatorname{rank}(A)=\operatorname{rank}(P A)=\operatorname{rank}(A Q)
$$

The above theorem shows that elementary operations will not change the rank. Thus, we can use elementary operations to simplify a matrix first and then use Theorem 1.18 to find the rank. 
Example 1.15. Find the rank of matrix $A$ in Example 1.5. We do a few column and row elementary operations to obtain

$$
\left(\begin{array}{lll}
2 & -1 & 3 \\
4 & -2 & 5 \\
2 & -1 & 4
\end{array}\right) \rightarrow\left(\begin{array}{lll}
0 & 1 & 3 \\
0 & 2 & 5 \\
0 & 1 & 4
\end{array}\right) \rightarrow\left(\begin{array}{ccc}
0 & 1 & 3 \\
0 & 0 & -1 \\
0 & 0 & 1
\end{array}\right) \rightarrow\left(\begin{array}{lll}
0 & 1 & 0 \\
0 & 0 & 0 \\
0 & 0 & 1
\end{array}\right)
$$

By Theorem 1.18, we can easily show that the rank of the last matrix above is 2 . Hence, the rank of $A$ is 2 .

\section{Block Matrix}

We can divide a matrix into blocks of submatrices. For example,

$$
A=\left(\begin{array}{cccc}
1 & 0 & 0 & 0 \\
0 & 1 & 0 & 0 \\
-1 & 2 & 1 & 0 \\
1 & 1 & 0 & 1
\end{array}\right)=\left(\begin{array}{ccccc}
1 & 0 & \vdots & 0 & 0 \\
0 & 1 & \vdots & 0 & 0 \\
\cdots & \cdots & \cdots & \cdots & \cdots \\
-1 & 2 & \vdots & 1 & 0 \\
1 & 1 & \vdots & 0 & 1
\end{array}\right)=\left(\begin{array}{cc}
I_{2} & 0 \\
A_{1} & I_{2}
\end{array}\right)
$$

where $A_{1} \equiv\left(\begin{array}{cc}-1 & 2 \\ 1 & 1\end{array}\right)$. Similarly, the following matrix is also divided into four blocks:

$$
B=\left(\begin{array}{ccccc}
1 & 0 & \vdots & 3 & 2 \\
-1 & 2 & \vdots & 0 & 1 \\
\cdots & \cdots & \cdots & \cdots & \cdots \\
1 & 0 & \vdots & 4 & 1 \\
-1 & -1 & \vdots & 2 & 0
\end{array}\right)=\left(\begin{array}{ll}
B_{11} & B_{12} \\
B_{21} & B_{22}
\end{array}\right)
$$

The advantage of this is that, in matrix manipulation, we can treat the blocks as numbers. In this example, we can treat $A$ and $B$ as $2 \times 2$ matrices. When all the matrix operations are feasible, the matrix operations can be applied direcly to the blocks. In the above example, one can easily verify that

$$
A B=\left(\begin{array}{cc}
B_{11} & B_{12} \\
A_{1} B_{11}+B_{21} & A_{1} B_{12}+B_{22}
\end{array}\right) .
$$

That is, by treating $A_{1}, I_{2}$ and $B_{i j}$ as numbers, we have

$$
\left(\begin{array}{cc}
I_{2} & 0 \\
A_{1} & I_{2}
\end{array}\right)\left(\begin{array}{ll}
B_{11} & B_{12} \\
B_{21} & B_{22}
\end{array}\right)=\left(\begin{array}{cc}
B_{11} & B_{12} \\
A_{1} B_{11}+B_{21} & A_{1} B_{12}+B_{22}
\end{array}\right) .
$$

In general,

$$
\left(\begin{array}{ccc}
A_{11} & \cdots & A_{1 l} \\
\vdots & & \vdots \\
A_{n 1} & \cdots & A_{n l}
\end{array}\right)\left(\begin{array}{ccc}
B_{11} & \cdots & B_{1 r} \\
\vdots & & \vdots \\
B_{l 1} & \cdots & B_{l r}
\end{array}\right)=\left(\begin{array}{cccc}
\sum_{k} A_{1 k} B_{k 1} & \cdots & \sum_{k} A_{1 k} B_{k r} \\
\vdots & & \vdots \\
\sum_{k} A_{n k} B_{k 1} & \cdots & \sum_{k} A_{n k} B_{k r}
\end{array}\right),
$$

where all $A_{i k} B_{k j}$ are feasible matrix multiplications. We, however, should not treat $A_{i k} B_{k j}$ as $B_{k j} A_{i k}$. That is, in matrix manipulation, we can treat matrix blocks as numbers as long as all 
the matrix multiplications are valid, but the order of the matrices must be kept in each multiplication.

Example 1.16. Let

$$
D \equiv\left(\begin{array}{cccccc}
a_{11} & \cdots & a_{1 k} & 0 & \cdots & 0 \\
\vdots & & \vdots & \vdots & & \vdots \\
a_{k 1} & \cdots & a_{k k} & 0 & \cdots & 0 \\
c_{11} & \cdots & c_{1 k} & b_{11} & \cdots & b_{1 r} \\
\vdots & & \vdots & \vdots & & \vdots \\
c_{r 1} & \cdots & c_{r k} & b_{r 1} & \cdots & b_{r r}
\end{array}\right)=\left(\begin{array}{cc}
A & 0 \\
C & B
\end{array}\right) .
$$

Assume that $A$ and $B$ are invertible. Let us find $D^{-1}$. Let

$$
D^{-1}=\left(\begin{array}{ll}
X_{11} & X_{12} \\
X_{21} & X_{22}
\end{array}\right)
$$

Then

$$
\left(\begin{array}{cc}
I_{k} & 0 \\
0 & I_{r}
\end{array}\right)=\left(\begin{array}{ll}
A & 0 \\
C & B
\end{array}\right)\left(\begin{array}{ll}
X_{11} & X_{12} \\
X_{21} & X_{22}
\end{array}\right)=\left(\begin{array}{cc}
A X_{11} & A X_{12} \\
C X_{11}+B X_{21} & C X_{12}+B X_{22}
\end{array}\right)
$$

which implies

$$
\left\{\begin{array}{l}
I=A X_{11} \\
A X_{12}=0 \\
C X_{11}+B X_{21}=0 \\
C X_{12}+B X_{22}=I
\end{array}\right.
$$

We then find $X_{11}=A^{-1}, X_{12}=0, X_{22}=B^{-1}$, and $X_{21}=-B^{-1} C A^{-1}$. That is,

$$
\left(\begin{array}{ll}
A & 0 \\
C & B
\end{array}\right)^{-1}=\left(\begin{array}{cc}
A^{-1} & 0 \\
-B^{-1} C A^{-1} & B^{-1}
\end{array}\right)
$$

In particular, when $C=0$, we have

$$
\left(\begin{array}{ll}
A & 0 \\
0 & B
\end{array}\right)^{-1}=\left(\begin{array}{cc}
A^{-1} & 0 \\
0 & B^{-1}
\end{array}\right)
$$

We can alternatively use Theorem 1.16 to find the inverse matrix. We have

$$
\left(\begin{array}{cccc}
A & 0 & I & 0 \\
C & B & 0 & I
\end{array}\right) \rightarrow\left(\begin{array}{cccc}
A & 0 & I & 0 \\
0 & B & -C A^{-1} & I
\end{array}\right) \rightarrow\left(\begin{array}{cccc}
I & 0 & A^{-1} & 0 \\
0 & I & -B^{-1} C A^{-1} & B^{-1}
\end{array}\right) .
$$

Hence, the inverse matrix is

$$
\left(\begin{array}{ll}
A & 0 \\
C & B
\end{array}\right)^{-1}=\left(\begin{array}{cc}
A^{-1} & 0 \\
-B^{-1} C A^{-1} & B^{-1}
\end{array}\right)
$$

\section{Eigenvalue and Diagonalization}

One important application of eigenvalues is to transform a matrix into a simple matrix. Consider transforming a square matrix $A \in \mathbb{R}^{n \times n}$ into a diagonal matrix as follows: there is a matrix $T$ such that 


$$
T^{-1} A T=D,
$$

where $D=\operatorname{diag}\left\{d_{1}, \ldots, d_{n}\right\}$ is a diagonal matrix with diagonal entries $d_{i}, i=1,2, \ldots n$, and zero elsewhere. Express $T$ as a sequence of column vectors $T=\left(\xi_{1}, \ldots, \xi_{n}\right)$. Then

$$
A\left(\xi_{1}, \ldots, \xi_{n}\right)=\left(\xi_{1}, \ldots, \xi_{n}\right)\left(\begin{array}{lll}
d_{1} & & \\
& \ddots & \\
& & d_{n}
\end{array}\right),
$$

implying

$$
\left(A \xi_{1}, \ldots, A \xi_{n}\right)=\left(d_{1} \xi_{1}, \ldots, d_{n} \xi_{n}\right)
$$

in turn implying

$$
A \xi_{i}=d_{i} \xi_{i}, \quad \text { for all } i .
$$

Hence, this expression is the key to identifying the diagonal matrix if it exists. Therefore, this expression is used to define eigenvalues.

For a square matrix $A \in \mathbb{R}^{n \times n}$, if there is a nonzero vector $x \in \mathbb{R}^{n}$ and a scalar $\lambda$ such that

$$
A x=\lambda x,
$$

then $\lambda$ is called a characteristic root or eigenvalue, and $x$ is called a characteristic vector or eigenvector. Call the following equation the characteristic equation of $A$ :

$$
|\lambda I-A|=0,
$$

and $|\lambda I-A|$ the characteristic polynomial. It turns out that this equation defines all the eigenvalues of $A$.

Theorem 1.19. $\lambda$ is an eigenvalue iff it satisfies the characteristic equation.

We have

$$
|\lambda I-A|=\lambda^{n}-\left(a_{11}+a_{22}+\cdots+a_{n n}\right) \lambda^{n-1}+\cdots+(-1)^{n} A .
$$

Example 1.17. For $A=\left(\begin{array}{ll}a & b \\ c & d\end{array}\right)$, we have

$$
\begin{aligned}
|\lambda I-A| & =\left|\begin{array}{cc}
\lambda-a & -b \\
-c & \lambda-d
\end{array}\right|=(\lambda-a)(\lambda-d)-b c=\lambda^{2}-(a+d) \lambda+a d-b c \\
& =\lambda^{2}-\operatorname{tr}(A) \lambda+|A| .
\end{aligned}
$$

This confirms the formula in (1.7).

Suppose that the eigenvalues of $A$ are $\lambda_{1}, \lambda_{2}, \ldots, \lambda_{n}$, which may repeat. Then

$$
|\lambda I-A|=\left(\lambda-\lambda_{1}\right) \cdots\left(\lambda-\lambda_{n}\right)=\lambda^{n}-\left(\lambda_{1}+\cdots+\lambda_{n}\right) \lambda^{n-1}+\cdots+(-1)^{n} \lambda_{1} \cdots \lambda_{n} .
$$

By comparing this with (1.7), we find

$$
\operatorname{tr}(A)=\lambda_{1}+\cdots+\lambda_{n}, \quad|A|=\lambda_{1} \cdots \lambda_{n} .
$$


Example 1.18. Given a matrix

$$
A=\left(\begin{array}{ll}
1 & 0 \\
1 & 2
\end{array}\right),
$$

let us use eigenvalues to simplify it (hopefully to a diagonal matrix). From the characteristic equation

$$
|\lambda I-A|=\left|\begin{array}{cc}
\lambda-1 & 0 \\
-1 & \lambda-2
\end{array}\right|=(\lambda-1)(\lambda-2)=0,
$$

we find two eigenvalues $\lambda_{1}=1$ and $\lambda_{2}=2$. For $\lambda_{1}=1$, from

$$
\left(\lambda_{1} I-A\right) x=\left(\begin{array}{cc}
\lambda_{1}-1 & 0 \\
-1 & \lambda_{1}-2
\end{array}\right) x=\left(\begin{array}{cc}
0 & 0 \\
-1 & -1
\end{array}\right) x=0,
$$

we find an eigenvector

$$
x_{1}=\left(\begin{array}{c}
1 \\
-1
\end{array}\right)
$$

For $\lambda_{2}=2$, from

$$
\left(\lambda_{2} I-A\right) x=\left(\begin{array}{cc}
\lambda_{2}-1 & 0 \\
-1 & \lambda_{2}-2
\end{array}\right) x=\left(\begin{array}{cc}
1 & 0 \\
-1 & 0
\end{array}\right) x=0,
$$

we find another eigenvector

$$
x_{2}=\left(\begin{array}{l}
0 \\
1
\end{array}\right)
$$

Let

$$
T \equiv\left(x_{1}, x_{2}\right)=\left(\begin{array}{cc}
1 & 0 \\
-1 & 1
\end{array}\right)
$$

Then

$$
A\left(x_{1}, x_{2}\right)=\left(A x_{1}, A x_{2}\right)=\left(\lambda_{1} x_{1}, \lambda_{2} x_{2}\right)=\left(x_{1}, x_{2}\right)\left(\begin{array}{cc}
\lambda_{1} & 0 \\
0 & \lambda_{2}
\end{array}\right) .
$$

That is,

$$
A T=T\left(\begin{array}{cc}
\lambda_{1} & 0 \\
0 & \lambda_{2}
\end{array}\right)
$$

We therefore have

$$
T^{-1} A T=\left(\begin{array}{cc}
\lambda_{1} & 0 \\
0 & \lambda_{2}
\end{array}\right)=\left(\begin{array}{ll}
1 & 0 \\
0 & 2
\end{array}\right)
$$

The transformation in the above example leads to a general theory of matrix transformation. Two square matrices $A, B \in \mathbb{R}^{n \times n}$ are similar if there is a square matrix $T \in \mathbb{R}^{n \times n}$ such that $T^{-1} A T=B$. We call this a similarity transformation. Such a transformation will not change the key characteristics of a matrix, including the determinant, rank, trace, and eigenvalues. A matrix $A$ is said to be diagonalizable if it is similar to a diagonal matrix.

Theorem 1.20. Eigenvectors belonging to different eigenvalues are linearly independent. 
Theorem 1.21. A square matrix $A \in \mathbb{R}^{n \times n}$ is diagonalizable iff it has $n$ linearly independent eigenvectors.

Example 1.19. Given a matrix

$$
A=\left(\begin{array}{ccc}
1 & 0 & 0 \\
-2 & 1 & 1 \\
-2 & 0 & 2
\end{array}\right)
$$

let us try to diagonalize it. From the characteristic equation

$$
|\lambda I-A|=\left|\begin{array}{ccc}
\lambda-1 & 0 & 0 \\
2 & \lambda-1 & -1 \\
2 & 0 & \lambda-2
\end{array}\right|=(\lambda-1)^{2}(\lambda-2)=0,
$$

we find two eigenvalues $\lambda_{1}=1$ and $\lambda_{2}=2$. For $\lambda_{1}=1$, from

$$
\left(\lambda_{1} I-A\right) x=\left(\begin{array}{ccc}
\lambda_{1}-1 & 0 & 0 \\
2 & \lambda_{1}-1 & -1 \\
2 & 0 & \lambda_{1}-2
\end{array}\right) x=\left(\begin{array}{ccc}
0 & 0 & 0 \\
2 & 0 & -1 \\
2 & 0 & -1
\end{array}\right) x=0,
$$

we find two linearly independent eigenvectors

$$
x_{1}=\left(\begin{array}{l}
0 \\
1 \\
0
\end{array}\right), \quad x_{2}=\left(\begin{array}{l}
1 \\
0 \\
2
\end{array}\right)
$$

We have deliberately chosen two orthogonal vectors. Orthogonal vectors must be linearly independent. For $\lambda_{2}=2$, from

$$
\left(\lambda_{2} I-A\right) x=\left(\begin{array}{ccc}
\lambda_{2}-1 & 0 & 0 \\
2 & \lambda_{2}-1 & -1 \\
2 & 0 & \lambda_{2}-2
\end{array}\right) x=\left(\begin{array}{ccc}
1 & 0 & 0 \\
2 & 1 & -1 \\
2 & 0 & 0
\end{array}\right) x=0,
$$

we find another eigenvector

$$
x_{3}=\left(\begin{array}{l}
0 \\
1 \\
1
\end{array}\right)
$$

Let

$$
T \equiv\left(x_{1}, x_{2}, x_{3}\right)=\left(\begin{array}{lll}
0 & 1 & 0 \\
1 & 0 & 1 \\
0 & 2 & 1
\end{array}\right)
$$

Then

$$
A\left(x_{1}, x_{2}, x_{3}\right)=\left(A x_{1}, A x_{2}, A x_{3}\right)=\left(\lambda_{1} x_{1}, \lambda_{1} x_{2}, \lambda_{2} x_{3}\right)=\left(x_{1}, x_{2}, x_{3}\right)\left(\begin{array}{ccc}
\lambda_{1} & 0 & 0 \\
0 & \lambda_{1} & 0 \\
0 & 0 & \lambda_{2}
\end{array}\right)
$$

That is,

$$
A T=T \cdot \operatorname{diag}\left\{\lambda_{1}, \lambda_{1}, \lambda_{2}\right\}
$$

We therefore have

$$
T^{-1} A T=\operatorname{diag}\left\{\lambda_{1}, \lambda_{1}, \lambda_{2}\right\}=\operatorname{diag}\{1,1,2\}
$$


In general, if

$$
|\lambda I-A|=\left(\lambda-\lambda_{1}\right)^{n_{1}} \cdots\left(\lambda-\lambda_{k}\right)^{n_{k}},
$$

where $\lambda_{i}, i=1, \ldots, k$, are all the distinct eigenvalues of $A$ and $n_{1}+\cdots+n_{k}=n$, then we should try to find $n_{i}$ linearly independent eigenvectors for each $\lambda_{i}$. If we succeed in doing so, this matrix is diagonalizable; otherwise it is not.

\section{Symmetric Matrix}

A complex number can be written as $z=a+b i$, where $a, b \in \mathbb{R}$ and $i \equiv \sqrt{-1}$. For each complex number $z=a+b i$, define its conjugate as $\bar{z} \equiv a-b i$. For any two complex numbers $x$ and $y$, we have

$$
\bar{x} \cdot y=\bar{x} \cdot \bar{y} .
$$

For a matrix $A=\left(a_{i j}\right)_{m \times n}$, define its conjugate as $\bar{A}=\left(\bar{a}_{i j}\right)_{m \times n}$. Then the above property also holds for matrix multiplication. That is, for any two matrices $A$ and $B$, we have

$$
\overline{A B}=\bar{A} \bar{B},
$$

as long as the matrix multiplication is valid.

A matrix $A$ is symmetric if $A^{\prime}=A$. A matrix $A$ is a real matrix if all its entries are real numbers.

Proposition 1.4. The eigenvalues of any real symmetric matrix are all real.

Two vectors $\alpha, \beta \in \mathbb{R}^{n}$ are orthogonal if $\alpha \cdot \beta=0$. A square matrix $T$ is orthogonal if $T^{\prime} T=I$, i.e., $T^{-1}=T^{\prime}$. A vector $\alpha$ is a unit vector if $\|\alpha\|=1$.

Proposition 1.5. $T \in \mathbb{R}^{n \times n}$ is orthogonal $\Leftrightarrow$ its column vectors are orthogonal unit vectors $\Leftrightarrow$ its row vectors are orthogonal unit vectors.

Proposition 1.6. If $A$ is a real symmetric matrix, then any two eigenvectors of different eigenvalues are orthogonal to each other.

Proposition 1.7. A collection of nonzero orthogonal vectors must be linearly independent.

Theorem 1.22. For any real symmetric matrix $A$, there exists an orthogonal matrix $T$ such that $T^{-1} A T$ is diagonal.

In the above theorem, the diagonal entries of $D \equiv T^{-1} A T$ are eigenvalues (not necessarily all distinct), and the column vectors of $T$ are the corresponding eigenvectors. 
Example 1.20. Consider the following second-degree equation:

$$
a_{11} x_{1}^{2}+2 a_{12} x_{1} x_{2}+a_{22} x_{2}^{2}+a_{1} x_{1}+a_{2} x_{2}+c=0 .
$$

Let

$$
A \equiv\left(\begin{array}{ll}
a_{11} & a_{12} \\
a_{12} & a_{22}
\end{array}\right), \quad a \equiv\left(\begin{array}{l}
a_{1} \\
a_{2}
\end{array}\right), \quad x \equiv\left(\begin{array}{l}
x_{1} \\
x_{2}
\end{array}\right) .
$$

By Theorem 1.22, there exists an orthogonal matrix $T$ and a diagonal matrix $D=\operatorname{diag}\left\{\lambda_{1}, \lambda_{2}\right\}$ such that $T^{-1} A T=D$. Let

$$
z \equiv\left(\begin{array}{l}
z_{1} \\
z_{2}
\end{array}\right) \equiv T^{-1}\left(\begin{array}{l}
x_{1} \\
x_{2}
\end{array}\right), \quad \beta \equiv\left(\begin{array}{l}
\beta_{1} \\
\beta_{2}
\end{array}\right) \equiv T a .
$$

Then if both $\lambda_{1} \neq 0$ and $\lambda_{2} \neq 0$,

$$
\begin{aligned}
x^{\prime} A x+a^{\prime} x+c & =x^{\prime} T T^{-1} A T T^{-1} x+a^{\prime} T T^{-1} x+c=z^{\prime} D z+\beta^{\prime} z+c \\
& =\lambda_{1} z_{1}^{2}+\lambda_{2} z_{2}^{2}+\beta_{1} z_{1}+\beta_{2} z_{2}+c \\
& =\lambda_{1}\left(z_{1}+\frac{\beta_{1}}{2 \lambda_{1}}\right)^{2}+\lambda_{2}\left(z_{2}+\frac{\beta_{2}}{2 \lambda_{2}}\right)^{2}+d,
\end{aligned}
$$

where $d$ is some constant. Let

$$
y_{i} \equiv z_{i}+\frac{\beta_{i}}{2 \lambda_{i}}, \quad i=1,2
$$

Then

$$
x^{\prime} A x+\alpha^{\prime} x+c=\lambda_{1} y_{1}^{2}+\lambda_{2} y_{2}^{2}+d=0
$$

By Proposition 1.4, we know that both $\lambda_{1}$ and $\lambda_{2}$ are real numbers. Hence, there are three possibilities: (1) $\lambda_{1}$ and $\lambda_{2}$ have the same sign; (2) $\lambda_{1}$ and $\lambda_{2}$ have different signs; and (3) either $\lambda_{1}$ or $\lambda_{2}$ is zero. By (1.10), the first two possibilities give the first two standard forms in the following:

Ellipse: $\quad \frac{y_{1}^{2}}{b_{1}^{2}}+\frac{y_{2}^{2}}{b_{2}^{2}}=1$,

Parabola: $\frac{y_{1}^{2}}{b_{1}^{2}}-\frac{y_{2}^{2}}{b_{2}^{2}}=1$ or $\frac{y_{2}^{2}}{b_{2}^{2}}-\frac{y_{1}^{2}}{b_{1}^{2}}=1$,

Hyperbola: $y_{1}^{2}=b_{2} y_{2}$ or $y_{2}^{2}=b_{1} y_{1}$. 


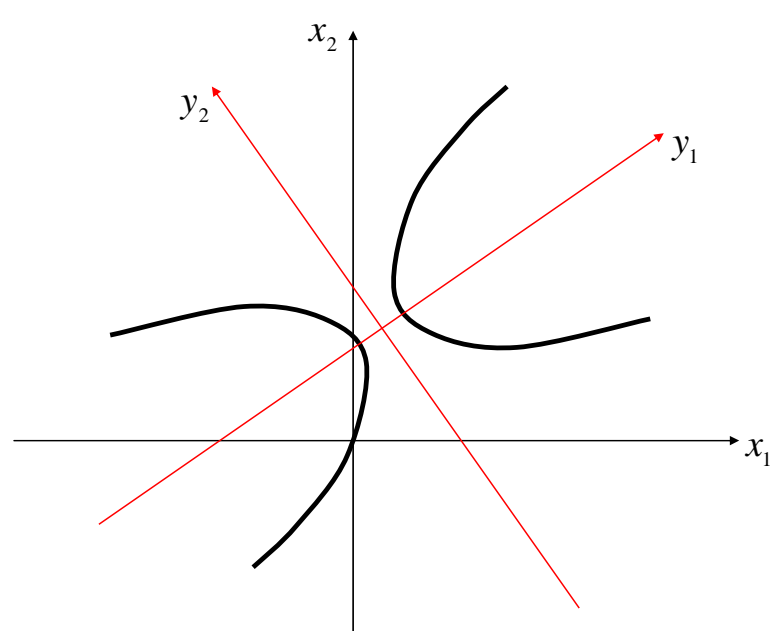

Figure 1.3. A Second-Degree Equation under Different Axis Systems

If one of $\lambda_{1}$ and $\lambda_{2}$ is zero, say $\lambda_{2}=0$, by the same derivation as in (1.9), we have

$$
x^{\prime} A x+\alpha^{\prime} x+c=\lambda_{1}\left(z_{1}+\frac{\beta_{1}}{2 \lambda_{1}}\right)^{2}+\beta_{2}\left(z_{2}+b\right),
$$

where $b$ is some constant. Let

$$
y_{1} \equiv z_{1}+\frac{\beta_{1}}{2 \lambda_{1}}, \quad y_{2} \equiv z_{2}+b .
$$

Then we have the third standard form in (1.11). ${ }^{1}$ In Figure 1.3, using the axis system for $\left(x_{1}, x_{2}\right)$, the two curves are represented by the equation in (1.8); but when the axis system for $\left(y_{1}, y_{2}\right)$ is used, the two curves are represented by $\frac{y_{1}^{2}}{b_{1}^{2}}-\frac{y_{2}^{2}}{b_{2}^{2}}=1$, which is much simpler. Changing the axis system corresponds precisely to a similarity transformation.

\section{Idempotent Matrix}

A real symmetric matrix $A$ is called an idempotent matrix if $A^{2}=A$.

Example 1.21. Given a full-rank tall matrix $H$, matrix $A \equiv H\left(H^{\prime} H\right)^{-1} H^{\prime}$ is symmetric and idempotent.

\section{Theorem 1.23.}

(1) The eigenvalues of an idempotent matrix are either 0 or 1.

(2) The only full-rank idempotent matrix is the identity matrix.

(3) The rank of an idempotent matrix is equal to its trace.

(4) Any idempotent matrix is positive semi-definite.

${ }^{1}$ See Matthews (1998, p.129) for details. 


\section{Notes}

Good references include Sydsæter et al. (2005, Chapter 1), Chiang (1984, Chapters 4 and 5), and Greene (1993, Chapter 2).

\section{Appendix}

\section{Proof of Theorem 1.4}

If $A$ is invertible, by definition, there exists a matrix $B$ such that $A B=I$. Hence,

$$
1=|I|=|A B|=|A| \cdot|B|,
$$

implying $|A| \neq 0$.

Conversely, if $|A| \neq 0$, we can construct a matrix $B$ by

$$
B \equiv \frac{1}{|A|} A^{*}
$$

For the matrix $A$, denote

$$
A_{i j} \equiv \text { the entry of } A \text { at the } i \text { th row and } j \text { th column. }
$$

By Theorem 1.3, we find

$$
\left(A A^{*}\right)_{i i}=\sum_{k=1}^{n} a_{i k} C_{i k}=|A|,
$$

and, for any $i$ and $j, i \neq j$, we find

$$
\left(A A^{*}\right)_{i j}=\sum_{k=1}^{n} a_{i k} C_{j k}=0 .
$$

Hence, $A A^{*}=|A| \cdot I_{n}$, implying that $A^{*} /|A|$ is the inverse matrix and thus $A$ is invertible.

\section{Proof of Corollary 1.1}

(1) If $A B=I$ and $C A=I$, then $C=C A B=I B=B$. That is, the inverse matrix is unique.

(2) For a square matrix $A$, if there is a matrix $B$ such that $A B=I$ or $B A=I$, we have $|A| \neq 0$ and hence, by Theorem 1.4, $A$ is invertible. By the uniqueness of an inverse matrix, we have $B=A^{-1}$. 


\section{Proof of Theorem 1.5}

By the definition of an inverse matrix, $A A^{-1}=I$ implies $A=\left(A^{-1}\right)^{-1}$, and $(A B)\left(B^{-1} A^{-1}\right)=$ $I$ implies $(A B)^{-1}=B^{-1} A^{-1}$.

\section{Proof of Theorem 1.6}

We prove only the third and fifth properties. The rest are obvious. To prove the third property, for a matrix $C$, denote

$$
C_{i j} \equiv \text { the entry of } C \text { at the } i \text { th row and } j \text { th column. }
$$

We have

$$
(A B)_{i j}^{\prime}=(A B)_{j i}=\sum_{k} A_{j k} B_{k i}=\sum_{k} B_{k i} A_{j k}=\sum_{k} B_{i k}^{\prime} A_{k j}^{\prime}=\left(B^{\prime} A^{\prime}\right)_{i j}
$$

Thus, $(A B)^{\prime}=B^{\prime} A^{\prime}$.

To prove the fifth property, since $A A^{-1}=I$, we have $\left(A^{-1}\right)^{\prime} A^{\prime}=I$, implying $\left(A^{-1}\right)^{\prime}=$ $\left(A^{\prime}\right)^{-1}$.

\section{Proof of Theorem 1.7}

We prove only the most important property: $\operatorname{tr}(A B)=\operatorname{tr}(B A)$. Since the entry at the ith row and jth column of $A B$ is $\sum_{k} a_{i k} b_{k j}$, we have

$\operatorname{tr}(A B)=\sum_{i}(A B)_{i i}=\sum_{i} \sum_{k} a_{i k} b_{k i}=\sum_{i} \sum_{k} b_{k i} a_{i k}=\sum_{k} \sum_{i} b_{k i} a_{i k}=\sum_{k}(B A)_{k k}=\operatorname{tr}(B A)$.

\section{Proof of Theorem 1.10}

Denote by $A$ a sequence of column vectors $A=\left(\alpha_{1}, \ldots, \alpha_{n}\right)$. The equation in (1.3) has a solution iff $d$ is a linear combination $\alpha_{1}, \ldots, \alpha_{n}$. Hence, if (1.3) has a solution, we have $\operatorname{rank}(A, d) \leq \operatorname{rank}(A)$, implying $\operatorname{rank}(A)=\operatorname{rank}(A, d)$. Conversely, if $\operatorname{rank}(A)=\operatorname{rank}(A, d)$, then $d$ must be a linear combination $\alpha_{1}, \ldots, \alpha_{n}$, implying that (1.3) has a solution.

\section{Proof of Theorem 1.16}

Suppose that there are elementary row operations $e_{1}, e_{2}, \ldots, e_{k}$ such that

$$
e_{1}(I) \cdot e_{2}(I) \cdots e_{k}(I)(A, I)=(I, B) .
$$


Then

$$
\left(e_{1}(I) \cdot e_{2}(I) \cdots e_{k}(I) A, e_{1}(I) \cdot e_{2}(I) \cdots e_{k}(I) I\right)=(I, B)
$$

implying

$$
e_{1}(I) \cdot e_{2}(I) \cdots e_{k}(I) A=I, \quad e_{1}(I) \cdot e_{2}(I) \cdots e_{k}(I) I=B .
$$

These in turn imply

$$
A^{-1}=e_{1}(I) \cdot e_{2}(I) \cdots e_{k}(I)=B
$$

\section{Proof of Theorem 1.17}

We apply the method of mathematical induction. First, this theorem is obviously true if $n=1$. Further, for any $n>1$, assume that any $(n-1) \times(n-1)$ invertible matrix is rowequivalent to the identity matrix. Given an invertible matrix $A=\left(a_{i j}\right)_{n \times n}$, at least one entry in the first column must be nonzero. We can move this nonzero entry $a_{i 1}$ to the first row by a third type of row operation. Since we can do this, we will simply assume $a_{11} \neq 0$. We can then use this nonzero entry to eliminate other entries in the first column using the second type of operations. That is, after some row operations, we are guaranteed to obtain

$$
A \rightarrow\left(\begin{array}{cccc}
1 & b_{12} & \cdots & b_{1 n} \\
0 & b_{22} & \cdots & b_{2 n} \\
\vdots & \vdots & & \vdots \\
0 & b_{n 2} & \cdots & b_{n n}
\end{array}\right)
$$

Since $A$ is invertible, $B$ must also be invertible, where

$$
B=\left(\begin{array}{ccc}
b_{22} & \cdots & b_{2 n} \\
\vdots & & \vdots \\
b_{n 2} & \cdots & b_{n n}
\end{array}\right)
$$

Since $B$ is invertible, by assumption, $B$ is row-equivalent to $I_{n-1}$, meaning that there is a matrix $P$ that is the product of a few elementary matrices such that $P B=I_{n-1}$. We have

$$
\left.\begin{array}{cccc}
1 & 0 & \cdots & 0 \\
0 & & & \\
\vdots & & P & \\
0 & & &
\end{array}\right)\left(\begin{array}{cccc}
1 & b_{11} & \cdots & b_{1 n} \\
0 & & & \\
\vdots & & B & \\
0 & & &
\end{array}\right)=\left(\begin{array}{cccc}
1 & b_{12} & \cdots & b_{1 n} \\
0 & & & \\
\vdots & & P B & \\
0 & & &
\end{array}\right)=\left(\begin{array}{cccc}
1 & b_{12} & \cdots & b_{1 n} \\
0 & & & \\
\vdots & & I_{n-1} & \\
0 & & &
\end{array}\right) .
$$

The last matrix in (1.12) is obviously row-equivalent to the identity matrix. Also, since, for any two square matrices $E_{1}$ and $E_{2}$, we have

$$
\left(\begin{array}{cc}
1 & 0 \\
0 & E_{1}
\end{array}\right)\left(\begin{array}{cc}
1 & 0 \\
0 & E_{2}
\end{array}\right)=\left(\begin{array}{cc}
1 & 0 \\
0 & E_{1} E_{2}
\end{array}\right)
$$

$\left(\begin{array}{ll}1 & 0 \\ 0 & P\end{array}\right)$ must be the product of a few elementary matrices. Hence, we have proven that any $n \times n$ invertible matrix is row-equivalent to the identity matrix. Therefore, by mathematical induction, any invertible matrix is row-equivalent to the identity matrix. 


\section{Proof of Theorem 1.19}

If $\lambda$ is an eigenvalue, then there exists an eigenvector $x$ such that $A x=\lambda x$, which implies $(\lambda I-A) x=0$. Thus, $|\lambda I-A|=0$. Conversely, if $|\lambda I-A|=0$, then there exists a nonzero $x$ such that $(\lambda I-A) x=0$, which implies $A x=\lambda x$.

\section{Proof of Theorem 1.21}

If there are $n$ independent eigenvectors $\alpha_{1}, \ldots, \alpha_{n}$ with corresponding eigenvalues $\lambda_{1}, \ldots, \lambda_{n}$, then

$$
\left(A \alpha_{1}, \ldots, A \alpha_{n}\right)=\left(\alpha_{1}, \ldots, \alpha_{n}\right)\left(\begin{array}{lll}
\lambda_{1} & & \\
& \ddots & \\
& & \lambda_{n}
\end{array}\right) .
$$

If we take $T \equiv\left(\alpha_{1}, \ldots, \alpha_{n}\right)$, we have

$$
T^{-1} A T=\operatorname{diag}\left\{\lambda_{1}, \ldots, \lambda_{n}\right\}
$$

Thus, $A$ is diagonalizable.

Conversely, if $A$ is diagonalizable to the matrix $D=\operatorname{diag}\left\{\lambda_{1}, \ldots, \lambda_{n}\right\}$ with $T^{-1} A T=D$ and $\alpha_{1}, \ldots, \alpha_{n}$ being the column vectors of $T$, then $\alpha_{1}, \ldots, \alpha_{n}$ are linearly independent and $A T=T D$, which implies $A \alpha_{i}=\lambda_{i} \alpha_{i}$ for all $i$. That is, $\alpha_{1}, \ldots, \alpha_{n}$ are eigenvectors.

\section{Proof of Proposition 1.4}

Let $A \xi=\lambda \xi$. Since $A=\bar{A}$ and $A=A^{\prime}$, we find

$$
\bar{\xi}^{\prime} A \xi=\lambda \bar{\xi}^{\prime} \xi \quad \text { and } \quad \bar{\xi}^{\prime} A \xi=\overline{(A \xi)^{\prime}} \xi=\overline{(\lambda \xi)^{\prime}} \xi=\bar{\lambda} \bar{\xi}^{\prime} \xi,
$$

implying $\bar{\lambda}=\lambda$.

\section{Proof of Proposition 1.5}

Express $T$ as a sequence of column vectors: $T=\left(\alpha_{1}, \ldots, \alpha_{n}\right)$. Then

$$
T^{\prime} T=\left(\begin{array}{c}
\alpha_{1}^{\prime} \\
\vdots \\
\alpha_{n}^{\prime}
\end{array}\right)\left(\alpha_{1}, \ldots, \alpha_{n}\right)=\left(\begin{array}{ccc}
\alpha_{1}^{\prime} \alpha_{1} & \cdots & \alpha_{1}^{\prime} \alpha_{n} \\
\vdots & & \vdots \\
\alpha_{n}^{\prime} \alpha_{1} & \cdots & \alpha_{n}^{\prime} \alpha_{n}
\end{array}\right) .
$$


Hence, $T^{\prime} T=I$ iff $\alpha_{i}^{\prime} \alpha_{i}=1$ for all $i$ and $\alpha_{i}^{\prime} \alpha_{j}=0$ for any $i \neq j$. That is, the column vectors of $T$ are orthogonal unit vectors.

Further, define the matrix $B$ as the transpose of $T$, i.e. $B \equiv T^{\prime}$. Since $T^{\prime} T=I$, we have $B^{\prime} B=I$, which by the above proof implies that the column vectors of $B$ are orthogonal unit vectors. The column vectors of $B$ are the row vectors of $T$.

\section{Proof of Proposition 1.6}

Given $A \xi=\lambda \xi$ and $A \eta=\delta \eta$ with $\lambda \neq \delta$, we have

$$
\eta^{\prime} A \xi=\lambda \eta^{\prime} \xi \quad \text { and } \quad \eta^{\prime} A \xi=(A \eta)^{\prime} \xi=\delta \eta^{\prime} \xi
$$

Hence, $\eta^{\prime} \xi=0$.

\section{Proof of Proposition 1.7}

Given a list of nonzero orthogonal vectors $\alpha_{1}, \ldots, \alpha_{n}$, consider

$$
\lambda_{1} \alpha_{1}+\cdots+\lambda_{n} \alpha_{n}=0 .
$$

If we multiply the above equation by $\alpha_{i}$, we get $\lambda_{i} \alpha_{i} \cdot \alpha_{i}=0$, implying $\lambda_{i}=0$. Hence, the above equation implies $\lambda_{1}=\cdots=\lambda_{n}=0$. Therefore, $\alpha_{1}, \ldots, \alpha_{n}$ are linearly independent.

\section{Proof of Theorem 1.23}

(1) By Theorem 1.22, there exists a diagonal matrix $D$ and an orthogonal matrix $T$ such that $T^{-1} A T=D$, where the diagonal entries of $D$ are the eigenvalues of $A$. Since $T^{-1} A T=D$ implies $T^{-1} A^{2} T=D^{2}$, we have $D=D^{2}$. Hence, any eigenvalue $\lambda$ must satisfy $\lambda=\lambda^{2}$, implying $\lambda=0$ or 1 .

Alternatively, since $A \xi=\lambda \xi$ and $A^{2} \xi=A(\lambda \xi)=\lambda^{2} \xi$ for the nonzero vector $\xi$, we must have $\lambda=\lambda^{2}$.

(2) If $A$ has full rank, since the rank does not change after a transformation, $D$ must have full rank too, implying $D=I$. Hence, $A=T D T^{-1}=I$.

(3) Since the rank and trace do not change after a transformation, we have

$$
\operatorname{rank}(A)=\operatorname{rank}(D) \quad \text { and } \quad \operatorname{trace}(A)=\operatorname{trace}(D) .
$$

Further, since $\operatorname{rank}(D)=\operatorname{trace}(D)$, we have $\operatorname{rank}(A)=\operatorname{trace}(A)$. 
(4) Since $D$ is positive semi-definite and semi-definiteness remains after a transformation, matrix $A$ must be positive semi-definite.

Alternatively, for any vector $x \in \mathbb{R}^{n}$, letting $y \equiv T^{\prime} x$ and $D=\operatorname{diag}\left\{d_{1}, \ldots, d_{n}\right\}$, we have

$$
x^{\prime} A x=x^{\prime}\left(T D T^{-1}\right) x=x^{\prime}\left(T D T^{\prime}\right) x=\left(T^{\prime} x\right)^{\prime} D\left(T^{\prime} x\right)=y^{\prime} D y=\sum_{i=1}^{n} d_{i} y_{i}^{2} \geq 0 .
$$

Hence, $A$ is positive semi-definite. 